\title{
A hysteresis model for timber joints with dowel-type fasteners
}

\author{
Matteo Izzi ${ }^{\mathrm{a}, \mathrm{b}, *}$, Giovanni Rinaldin $^{\mathrm{c}}$, Andrea Polastri ${ }^{\mathrm{a}}$, Massimo Fragiacomo ${ }^{\mathrm{d} \text {,a }}$ \\ a National Research Council of Italy - Trees and Timber Institute (CNR IVALSA), Via Biasi 75, 38010 San Michele all'Adige, Italy \\ ${ }^{\mathrm{b}}$ Department of Engineering and Architecture, University of Trieste, Piazzale Europa 1, 34127 Trieste, Italy \\ ${ }^{\mathrm{c}}$ Department of Architecture, Design and Urban Planning, University of Sassari, Piazza Duomo 6, 07041 Alghero, Italy \\ d Department of Civil, Construction-Architectural and Environmental Engineering, University of L'Aquila, Via Gronchi 18, 67100 L'Aquila, Italy
}

\section{A R T I C L E I N F O}

\section{Keywords:}

Cross-Laminated Timber

Annular-ringed shank nail

Steel-to-timber joint

Non-linear modelling

Hysteretic behaviour

\begin{abstract}
A B S T R A C T
Predicting the mechanical behaviour and the failure mechanism of timber joints with dowel-type fasteners requires consideration of several factors, including the geometrical and mechanical properties of the metal fastener, the physical properties of timber and the interaction between such elements. This paper proposes a numerical model where a joint is schematized as an elasto-plastic beam in a non-linear medium with a compression-only behaviour. Unlike the differential approach adopted by most of the hysteresis models published in literature, this model predicts the load-displacement response using simple mechanical relationships and basic input parameters. Furthermore, the model is capable of reproducing the effect of the cavity formed around the fastener by timber crushing, and simulates the hysteretic behaviour and the energy dissipation under cyclic conditions. Shear tests are reproduced on nailed steel-to-timber joints in Cross-Laminated Timber and results are compared to the experimental test data obtained on similar single fastener joints. Simulations lead to accurate predictions of both the mechanical behaviour (initial stiffness, maximum load-carrying capacity, global shape of the loading curve and of the hysteresis cycles) and the total energy dissipation observed in the tests.
\end{abstract}

\section{Introduction}

Timber structures are made of $1 \mathrm{D}$ (e.g. beams and studs) and 2D elements (e.g. walls and floors) fastened together with mechanical joints and connection systems that transmit the lateral shear and tension loads. Due to the high strength-to-weight ratio of timber and the connections capacity to resist the load with ductile deformations and little impairment of strength, these structures showed satisfactory performances in seismic conditions [1-5].

Mechanical joints in timber structures are assembled using doweltype fasteners (nails, staples, screws, bolts, and dowels). Their loaddisplacement response depends on several factors, including the yielding moment and the withdrawal behaviour of the fastener, the embedment behaviour of timber, and the interaction between fastener shank and timber.

Eurocode 5 [6] defines the load-carrying capacity of joints with dowel-type fasteners according to the European Yield Model (EYM), originally proposed by Johansen [7]. The rope effect is included into the design equations and some limiting factors, expressed at a maximum percentage of the lateral dowel capacity of the joint, are introduced to avoid relying on the withdrawal of the fastener. Since the EYM is developed based on a plastic limit analysis, it is suitable to determine collapse loads, while it cannot be used to predict the loaddisplacement response under monotonic or cyclic conditions.

Alternative calculation methods were proposed since the early ' $50 \mathrm{~s}$ to overcome the limitations of the EYM. Ivanov [8] developed an empirical quadratic equation to relate the strength of a nailed joint to its displacement. Mack [9] proposed a calculation model where the loaddisplacement response is determined as product of a series of independent factors. Finally, Kuenzi [10] reproduced a single- or doubleshear joint as a beam on an elastic foundation. Using a fourth-order differential equation, this model estimated the shear and deflection at any point of the joint; however, it had limited applications since it could be used only in the elastic range of the loading curve.

In recent years, many research projects focused on developing hysteresis models able to predict both the elastic and inelastic response of joints with dowel-type fasteners. Three approaches were followed: the first one aimed at improving the results obtained with the beam on an elastic foundation, by reproducing the embedment behaviour of timber with non-linear springs [11-14]. In the second approach, the non-linear response of the joint was concentrated into hysteretic springs or simple elasto-plastic systems [15-21]. Generally, such models were used to analyse the behaviour of light-frame shear walls or full-scale timber structures, i.e. situations where computationally efficient

\footnotetext{
* Corresponding author at: CNR IVALSA, Via Biasi 75, 38010 San Michele all'Adige, Italy.

E-mail address: izzi@ivalsa.cnr.it (M. Izzi).
} 
algorithms are needed. Finally, the third one aimed at developing accurate schematizations of the joints where the actual material properties are assigned to the elements of the models [22-26]. Regarding this third approach, it should be noticed that significant efforts have been devoted to develop material models capable of predicting the mechanical behaviour and the failure mechanisms of timber [27-29].

This paper proposes a numerical model able to predict the loaddisplacement response and failure mechanisms of timber joints with dowel-type fasteners. The model is capable of reproducing the effect of the cavity formed around the fastener by timber crushing, allowing the prediction of the hysteretic behaviour and the energy dissipation under cyclic conditions. The joint is schematized as an elasto-plastic beam embedded in a non-linear medium with a compression-only behaviour. Unlike the differential approach adopted by most of the hysteresis models published in literature, this model adopts simple mechanical relationships and basic input parameters to reproduce the response of the steel and timber components of the joint. In addition to the geometrical data, the yielding moment and the withdrawal behaviour of the fastener, and the embedment behaviour of timber are the required input parameters. Such input values can be derived either from tests (carried out, e.g., according to EN 409 [30], EN 383 [31] and EN 1382 [32]) or from the experimental results and analytical formulas published in literature (e.g. [33-35]).

The model proposed in this contribution can be used to predict the response of several types of joints. In fact, with minor modifications on the boundary conditions, the response of a timber-to-timber, a steel-totimber, and a slotted-in steel plate joint can be simulated (Fig. 1). Results obtained on single fastener joints can be employed to analyse systems where many of these elements are present. For instance, the predicted load-displacement response of a nailed joint can be concentrated into a non-linear hysteretic spring and used to investigate the mechanical behaviour of a metal connector (e.g. an angle bracket or a hold-down) or of a light-frame shear-wall.

Shear tests are reproduced on steel-to-timber joints with annularringed shank nails in Cross-Laminated Timber (CLT). Firstly, the mechanical behaviour of the nailed joints is validated by comparing the numerical predictions of the lateral dowel capacity with the analytical values assessed using a model proposed by Hilson [36]. The load-displacement response under monotonic conditions is subsequently investigated by reproducing typical single fastener joints shear tests in parallel and perpendicular to the face lamination of a CLT panel. Finally, the hysteretic behaviour and the energy dissipation under seismic conditions are analysed by reproducing cyclic shear tests. Numerical results are compared to recently obtained test data of nailed steel-totimber joints in CLT, and differences are discussed. All the simulations are performed using ABAQUS software package [37].

\section{Model description}

The numerical model proposed herein schematizes a single fastener joint as an elasto-plastic beam in a non-linear medium with a compression-only behaviour. A key feature of this modelling technique is the presence of non-linear springs capable of reproducing the hysteretic behaviour of the steel and timber components of the joint (Fig. 1). In this study, these springs are simulated with User Element Subroutines (UELs) taken from Rinaldin et al. [20,38]. A preliminary version of this model was presented by Rinaldin [39].

\subsection{Fastener schematization}

The fastener is modelled as an elastic system with concentrated plasticity. The shank is discretized into a series of elastic beams interconnected with hinges (see the schematics of the numerical model in Fig. 1 and the close-up given in Fig. 2). The presence of non-linear rotational springs at the hinge locations ensures the transmission of the bending moment between adjacent beams. Furthermore Moreover,
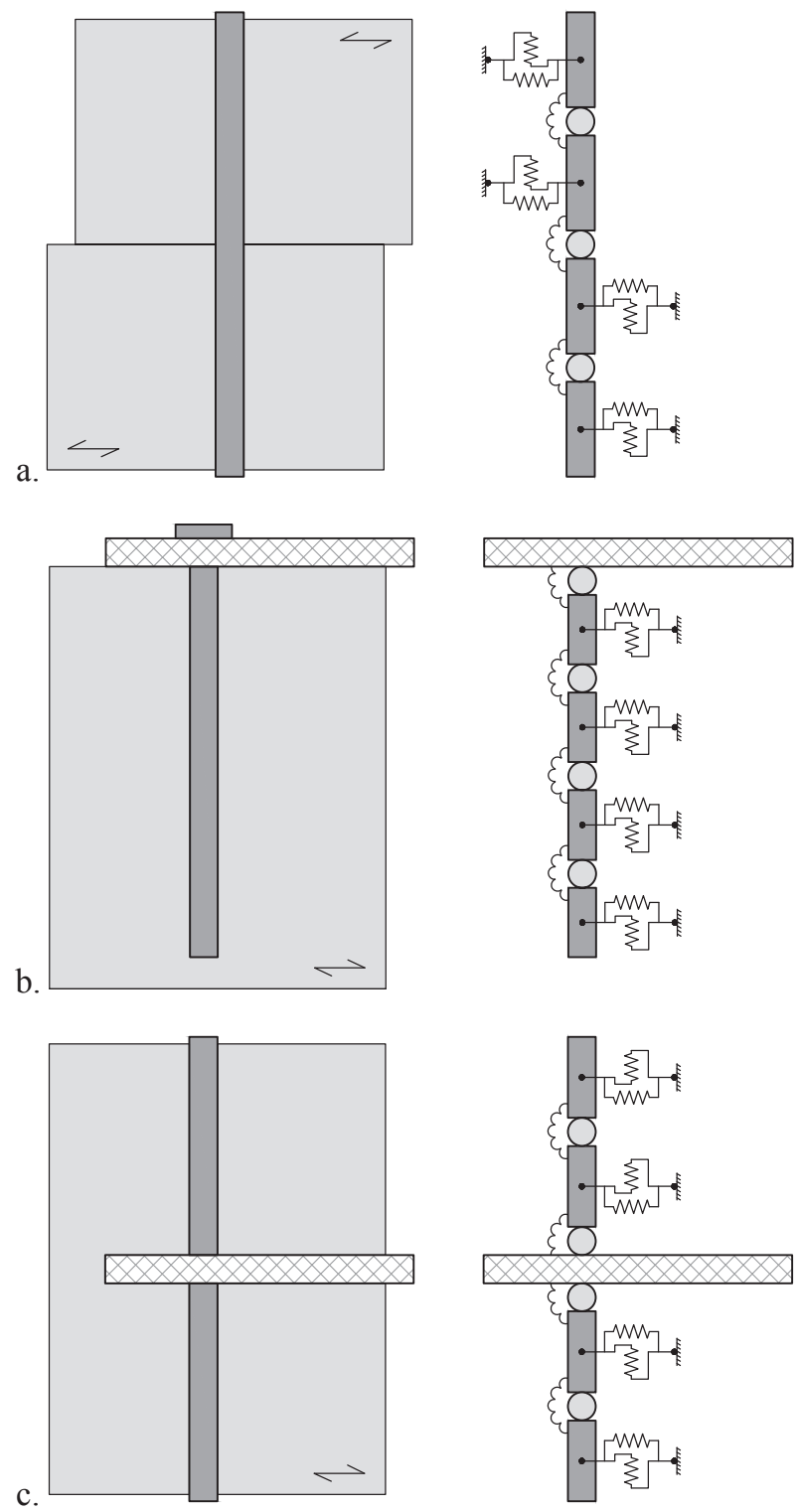

Fig. 1. Schematics of a (a) timber-to-timber, (b) steel-to-timber, and (c) slotted-in steel plate joint (left, according to Eurocode 5 [6]; right, according to the proposed numerical model).

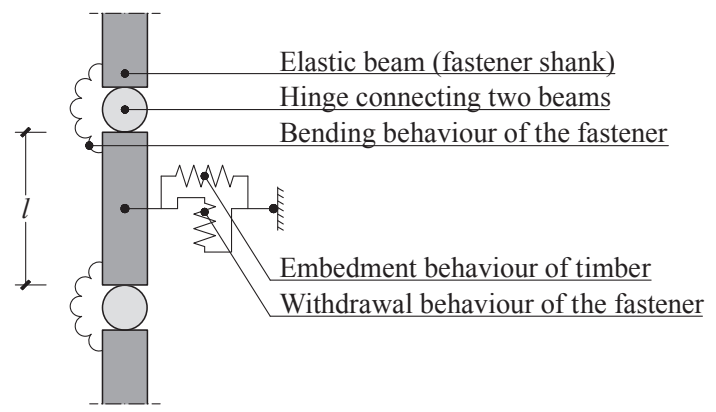

Fig. 2. Close-up of the numerical model, with description of its components.

those springs control the bending behaviour once the fastener reaches its plastic deformed configuration.

The bending behaviour of the fastener is simulated as follows: at small displacement amplitudes (Fig. 3a), the deflections are due to the bending deformations of the beams, while the springs transmit the bending moment and prevent any rigid body rotation. Once the bending 


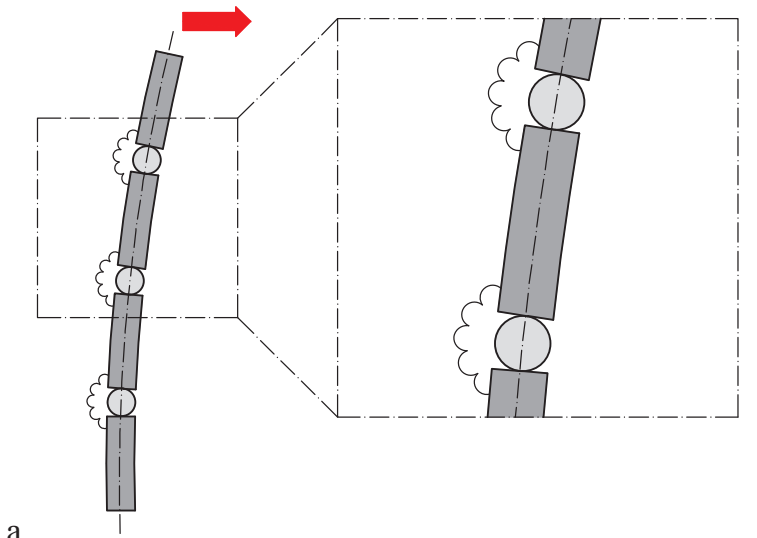

a.

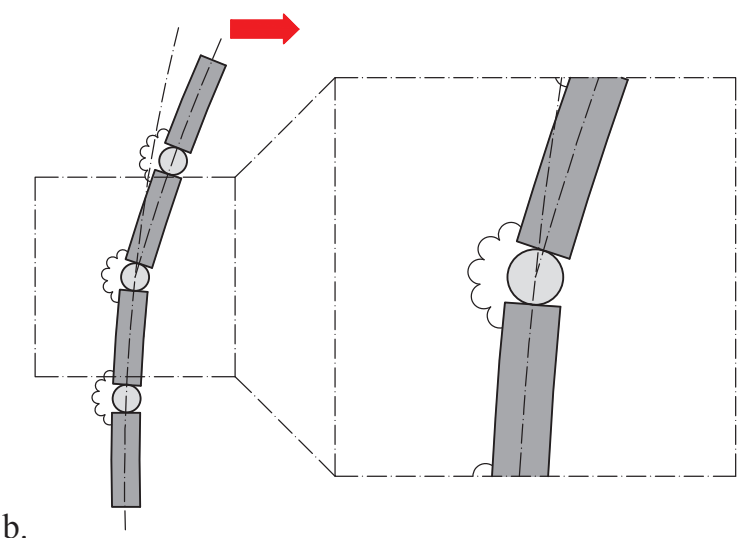

Fig. 3. Bending behaviour of the fastener: (a) at small displacement amplitudes, when the deflections are due to the bending deformations of the elastic beams, and (b) when the moment on the shank reaches the yielding value and the non-linear behaviour of the rotational springs is activated.

moment reaches the yielding value (Fig. 3b), the non-linear behaviour of the rotational springs is activated. In such situation, the moment taken by those springs is kept constant regardless the rotation, and a plastic behaviour is simulated.

The hinges are modelled as pins using ABAQUS 'multi-point constraints' (MPC) [37]. The length of each beam $l$ depends on the fastener discretization and is equal to the ratio of the penetration depth to the number of beams. The mechanical behaviour of the beams is assumed elastic, although two constitutive laws are adopted in the axial and transversal direction. A very stiff elastic behaviour is adopted in the axial direction, with stiffness $K_{\text {axial }}$ conventionally set to 10 times the actual axial stiffness of the beams:

$K_{\text {axial }}=10 \frac{E A}{l}$

In Eq. (1), the symbol $E$ denotes the stiffness of the steel material ( $E=210 \mathrm{GPa}$ ), while $A$ represents the cross-section area of the shank ( $A=\pi d^{2} / 4$, being $d$ the diameter of the shank). Furthermore, an elastic behaviour is considered in the transversal direction, with bending stiffness $K_{\text {bending }}$ defined as shown in Eq. (2). In the equation below, $J$ is the moment of inertia of the beam cross-section (with $J=\pi d^{4} / 4$ ).

$K_{\text {bending }}=\frac{E J}{l}$

The uncoupled behaviour between axial and transversal direction is necessary to keep the fastener length constant once the joint reaches its plastic deformed configuration and is implemented in the analyses by defining the cross-section properties using ABAQUS 'beam general section' command [37].

As mentioned above, each rotational spring is connected to the ends

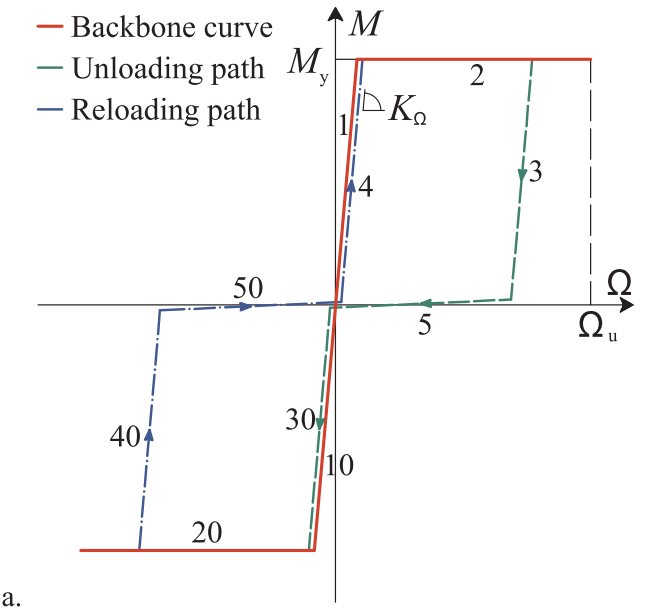

a.

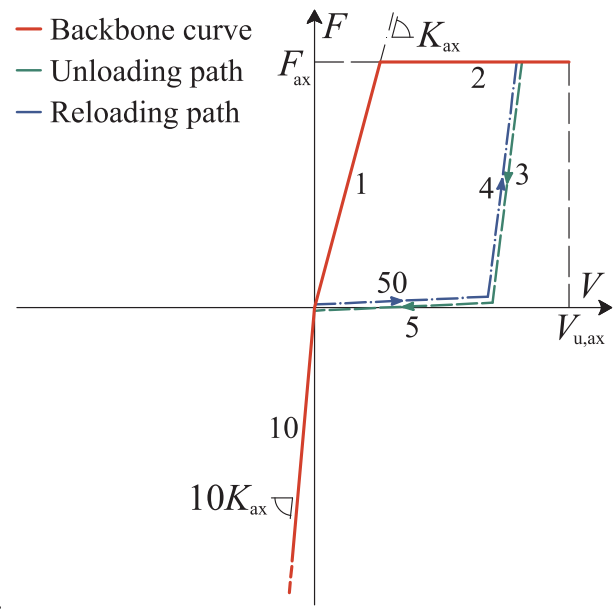

b.

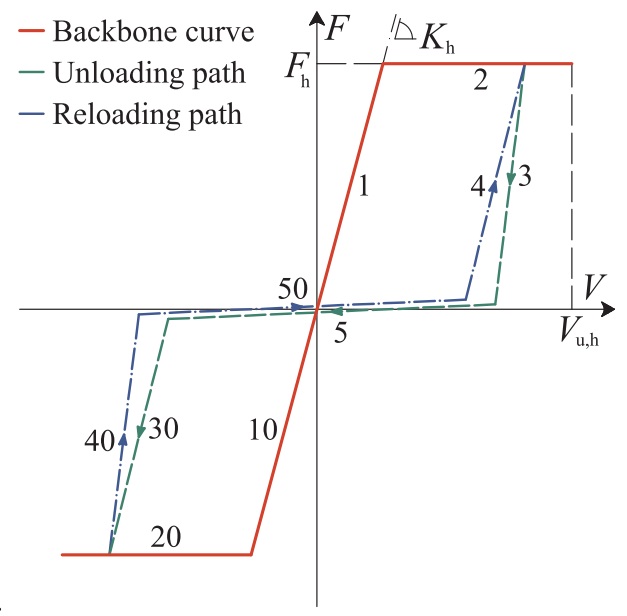

Fig. 4. Piecewise-linear laws schematizing: (a) the bending behaviour and (b) the withdrawal behaviour of the fastener, and (c) the embedment behaviour of timber (adapted from Rinaldin et al. $[20,38])$.

of two consecutive beams and has an elasto-plastic moment-rotation relationship with elastic stiffness $K_{\Omega}$ and yielding moment $M_{\mathrm{y}}$. Theoretically, the moment-rotation relationship implemented into the rotational springs should be rigid-plastic. However, a rigid behaviour cannot be modelled using spring elements and an elasto-plastic law with very high elastic stiffness is used instead. In this study, the stiffness $K_{\Omega}$ is determined by assuming that the yielding moment is attained at a conventional rotation of $0.1^{\circ}$. The inelastic branch has a perfectly plastic behaviour until the ultimate rotation $\Omega_{\mathrm{u}}$, which is set to $45^{\circ}$ 
[30].

The piecewise-linear law governing the rotational springs is displayed in Fig. 4a; the backbone curve (composed of branches \#1, \#2, $\# 10$, and $\# 20$ ) is obtained as discussed above. If the springs are unloaded from a positive rotation, branch \#3 is followed. On the contrary, branch \#40 is followed if the springs are unloaded from a negative rotation. The slope of branches \#3 and \#30 is the same of \#1 and \#10 while the load at the transition point between branches \#3 and \#5 is conventionally set to $2 \%$ of the load attained on the backbone curve at the current hysteresis cycle. Finally, the transition point between \#5 and \#30 is located in the origin of the axis.

The presence of supplementary boundary conditions is taken into account in the model by introducing additional spring elements. In a steel-to-timber joint, the clamping of the fastener cap to the metal plate is simulated with an additional spring located between the upper beam and the metal member to which the cap is connected (Fig. 1b). In a slotted-in steel plate joint, the clamping of the fastener shank to the plate is simulated with two additional spring elements located between the metal member and the connected beams (Fig. 1c).

\subsection{Withdrawal behaviour of the fastener}

The withdrawal behaviour of the fastener is simulated with a set of non-linear hysteretic springs uniformly distributed along the fastener shank (see general schematics in Fig. 1). Each spring is connected to a master node, located in the centre of the beam where it is attached, and to a fixed point of the surrounding space (see close-up in Fig. 2).

The withdrawal spring has a non-symmetric load-displacement relationship. If loaded in tension, an elasto-plastic behaviour is adopted with elastic stiffness $K_{\mathrm{ax}}$ (in this study referred to as 'withdrawal stiffness') and withdrawal capacity $F_{\mathrm{ax}}$. The inelastic branch has a plastic behaviour until the ultimate displacement $V_{\mathrm{u}, \mathrm{ax}}$, conventionally set to $10 \mathrm{~mm}$. If the spring is loaded in compression, based on the schematics of Fig. 1, the fastener cannot penetrate into the timber embedment and a very stiff elastic behaviour is adopted (conventionally set to 10 times the withdrawal stiffness $K_{\mathrm{ax}}$ ).

The withdrawal stiffness of a spring and its capacity are defined based on the fastener shank discretization, as shown in Eqs. (3) and (4).

$K_{\mathrm{ax}}=\frac{K_{\mathrm{ax}, \text { fastener }}}{n}$

$F_{\mathrm{ax}}=\frac{F_{\mathrm{ax}, \text { fastener }}}{n}$

In the equations above, $n$ is the number of beam elements used to discretize the shank, while $K_{\mathrm{ax} \text {,fastener }}$ and $F_{\mathrm{ax}}$,fastener represent the withdrawal stiffness (measured in $\mathrm{N} / \mathrm{mm}$ ) and capacity (expressed in $\mathrm{N}$ ) of the entire fastener, respectively.

The piecewise-linear law of the withdrawal springs should be specifically calibrated for the analysed joint. For instance, Fig. 4b shows the typical hysteretic behaviour of a steel-to-timber joint under withdrawal loads. The backbone curve (composed of branches \#1, \#2, and $\# 10$ ) is obtained as discussed above. Once extracted, the steel plate is not able to push the fastener backwards inside the timber element. Therefore, under cyclic loading conditions, branch \#3 simulates the sudden reduction in load-carrying capacity due to the gap formed between the metal plate and the fastener cap. If the spring is reloaded (branch \#4), the bearing mechanism under withdrawal loads is not activated until the gap between the metal plate and the fastener cap is closed. According to this behaviour, the slope of branches \#3 and \#4 is conventionally set to $5 K_{\mathrm{ax}}$.

\subsection{Embedment behaviour of timber}

The embedment behaviour of timber in compression is simulated with a set of non-linear hysteretic springs uniformly distributed along the fastener shank (Fig. 1). Each spring is connected to a master node located in the centre of the corresponding beam and to a fixed point of the surrounding space (Fig. 2). To limit the computational effort, the embedment behaviour in front of and behind a beam is simulated with a unique spring.

Each non-linear spring has an elasto-plastic load-displacement relationship with elastic stiffness $K_{\mathrm{h}}$ (in this study referred to as 'embedment stiffness') and embedment capacity $F_{\mathrm{h}}$. The inelastic branch simulates the crushing of the fibres underneath the fastener and the associated densification of timber in the contact area [40]; it has a plastic behaviour until the ultimate displacement $V_{\mathrm{u}, \mathrm{h}}$, conventionally set to $15 \mathrm{~mm}$.

The embedment stiffness of a non-linear spring and its capacity depend on the fastener shank discretization and are proportional to the tributary area of each beam $(d \cdot l)$. In this study, those properties are determined using a foundation modelling approach, as shown in Eqs. (5) and (6).

$K_{\mathrm{h}}=k_{\mathrm{h}}(d \cdot l)$

$F_{\mathrm{h}}=f_{\mathrm{h}}(d \cdot l)$

In the equations above, $k_{\mathrm{h}}$ is the modulus of subgrade reaction (measured in $\mathrm{MPa} / \mathrm{mm}$ ) and $f_{\mathrm{h}}$ is the embedment strength of timber (expressed in MPa). Usually, the embedment behaviour of timber depends on the relative orientation of the applied load with respect to the grain direction. Fasteners loaded parallel to the grain exhibit a high initial stiffness and a plastic plateau, while fasteners loaded perpendicular to the grain are less stiff at low loads but have a continuous load increase before failure [41-44]. However, according to Zhou and Guan [45] and Hong and Barrett [24], the embedment capacity is independent of the load-to-grain angle if the fastener diameter is smaller than or equal to $8 \mathrm{~mm}$.

The model is also capable of reproducing the effects of a layered timber embedment. For instance, the layup of a CLT element can be simulated by defining specific values of $K_{\mathrm{h}}$ and $F_{\mathrm{h}}$, depending on the load-to-grain direction and the spring position along the fastener shank.

The piecewise-linear law governing the non-linear springs is displayed in Fig. 4c; the backbone curve (composed of branches \#1, \#2, $\# 10$, and \#20) is obtained as discussed above. If the springs are unloaded from a positive displacement, branch $\# 3$ is followed until the attainment of zero load. On the contrary, branch \#40 is followed if the unloading starts from a negative displacement. Branches \#5 and \#50 simulate the gap between the supporting medium and the fastener, caused by the cavity formed by timber crushing. Finally, branches \#30 and \#4 take to the opposite side of the backbone curve and simulate the embedment behaviour at increased displacement levels, when the shank comes again into contact with the surrounding timber. The slopes of branches \#3 and \#4 are defined based on the tests performed by Chui and $\mathrm{Ni}$ [46] and are expressed as functions of the embedment stiffness $K_{\mathrm{h}}$ : the first one is set to $5 K_{\mathrm{h}}$ while the latter one to $2.5 K_{\mathrm{h}}$. The transition point between branches \#3 and \#5 (and between \#50 and \#4) depends on the load reached on the backbone curve and is updated at every hysteresis cycle.

\subsection{Embedding of the timber fibres into the fastener thread}

Laterally loaded joints assembled with profiled fasteners exhibit embedding of the timber fibres into the thread of the shank. According to Domínguez et al. [47], three stages can be identified. The first one takes place at small displacement amplitudes, when the bearing mechanism is associated to bending of the fastener shank together with compression of the timber fibres. In such situation, the contact between shank and timber occurs only at the pitch of the thread, on a significantly smaller surface compared to the case of smooth fasteners (Fig. 5a). The second stage takes place when the joint attains its lateral dowel capacity. Here, the deformed fastener has no displacement along 

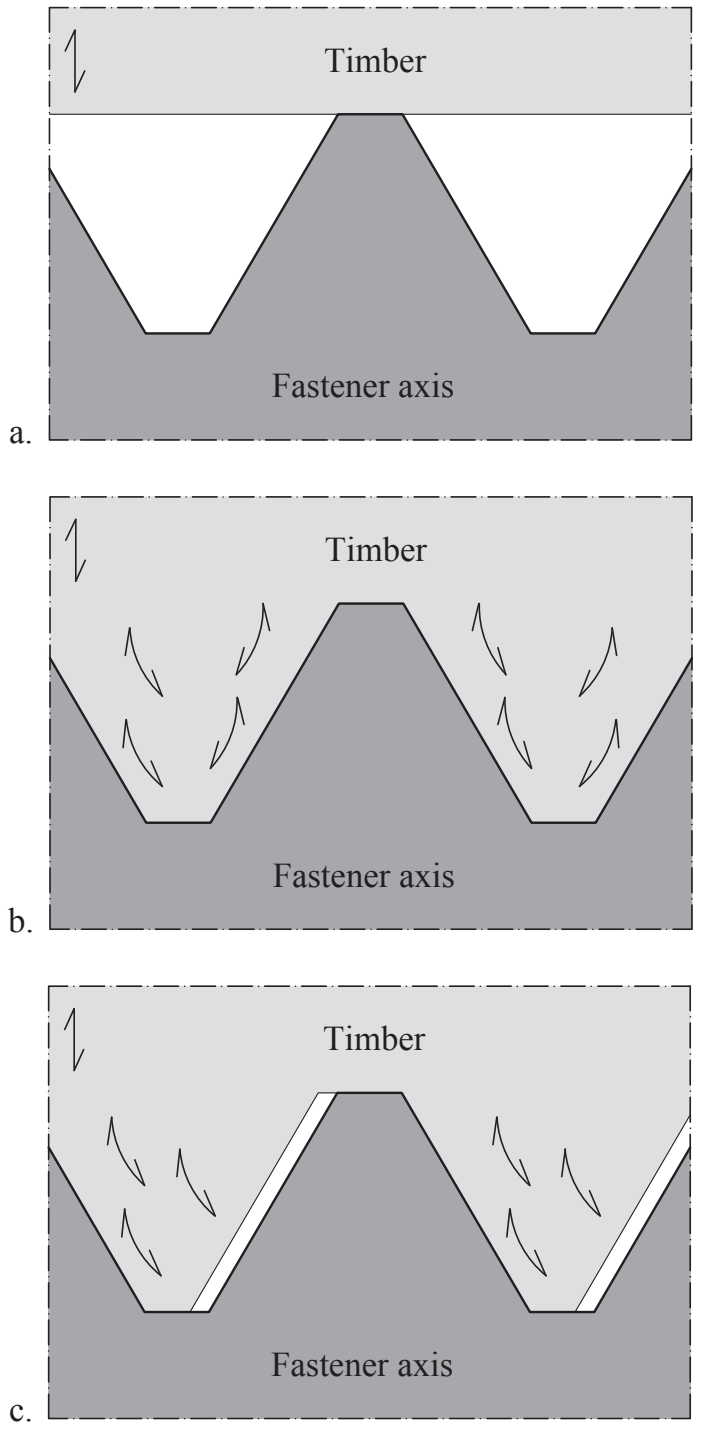

Fig. 5. Embedding of the timber fibres into the fastener thread: (a) prior to load application, (b) crushing without displacement along the fastener axis, and (c) crushing with displacement along the fastener axis (adapted from Domínguez et al. [47]).

its axis and the crushed timber embeds in the thread of the shank (Fig. 5b). Finally, the third stage takes place when the joint is subjected to higher lateral displacements and the fastener slips over the crushed timber. In such situation, the axial displacement of the fastener causes one side of the timber inside the thread to be subjected to embedding against a flank of the thread while the other side loses contact (Fig. 5c). Consequently, the timber fibres surrounding the thread resist the slip of the fastener and increase its withdrawal stiffness.

As recommended also by Domínguez et al. [47], the model simulates the embedding of the timber fibres into the fastener thread by increasing the stiffness of the withdrawal springs. In particular, the same authors suggest increasing the withdrawal stiffness so that the maximum withdrawal capacity of the fastener is attained once the axial displacement exceeds values around the pitch of the thread.

\section{Model calibration}

The model proposed in this paper is calibrated considering a nailed steel-to-timber joint in CLT (Fig. 6). The model is assembled according to the schematic shown in Fig. 1b. Analyses considered typical annularringed shank nails with diameter $d=4.0 \mathrm{~mm}$, threaded length $l_{\mathrm{thr}}=44 \mathrm{~mm}$ and pointside penetration depth $t_{1}=54 \mathrm{~mm} \mathrm{[48]} \mathrm{(Fig.} \mathrm{7).}$

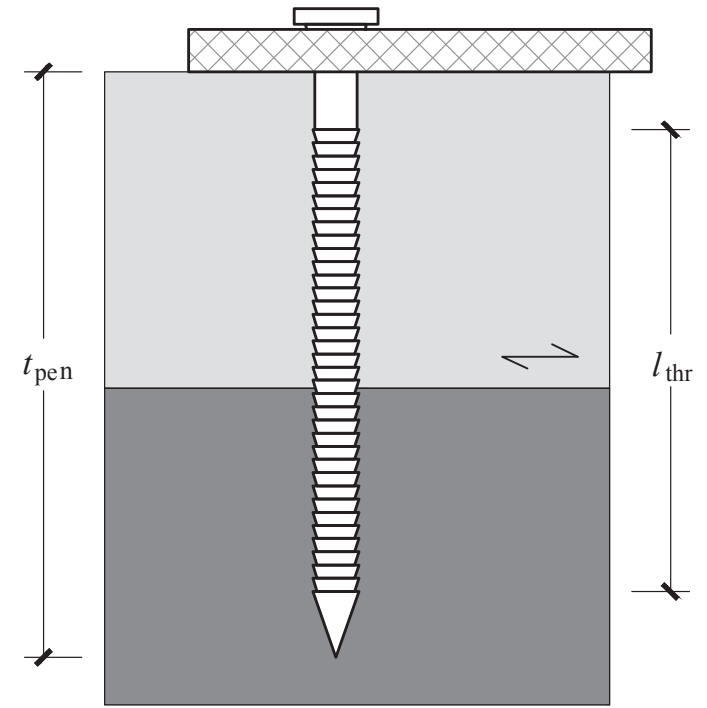

Fig. 6. Schematic of the nailed steel-to-timber joint considered in the simulations.

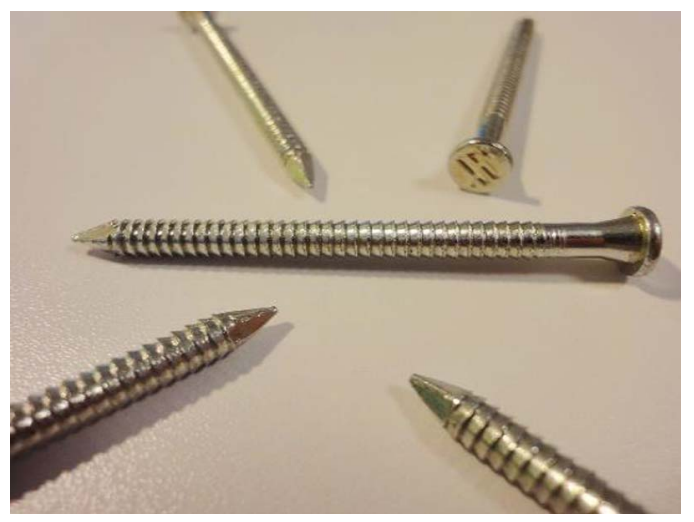

Fig. 7. Annular-ringed shank nails (reproduced from Izzi et al. [49]).

As mentioned in the introduction, the input parameters required by the model can be assessed using either experimental approaches or the information available in literature. In the first case, specific tests should be performed according to the reference standards (EN 409 [30], EN 383 [31] and EN 1382 [32]), leading to an accurate definition of those properties. However, this method is time consuming and requires the execution of a large number of tests. On the contrary, in the second case, the input parameters are determined based on the experimental results and general formulas published in literature. One of the major advantages of this second method, which was adopted in the following study, is the possibility of extending the test results available for a joint to other configurations of technical interest (e.g., varying the diameter of the fastener, the density of timber, and the load-to-grain angle).

\subsection{Strength capacities}

In the following, analyses are performed by defining the yielding moment of the nail $M_{\mathrm{y}}$ as the plastic moment capacity of the circular cross-section (Eq. (7)). The yielding strength of the nail $f_{\mathrm{y}}$, which is an input parameter needed by the model, is determined according to Sandhaas and Mergny [33] as shown in Eq. (8).

$M_{\mathrm{y}}=\frac{1}{6} f_{\mathrm{y}} d^{3}$

$f_{\mathrm{y}}=1154 d^{-0.29}$

Similarly, the withdrawal capacity of the nail $F_{\mathrm{ax}}$ is defined 
according to Eq. (9), following an analytical formula proposed by Bla $\beta$ and Uibel [34]. In the equation below, $l_{\text {thr }}$ denotes the threaded length of the shank and $\rho$ the average density of timber.

$F_{\mathrm{ax}}=0.155 \rho^{0.8} d^{0.6} l_{\mathrm{thr}}$

Finally, the embedment strength of timber $f_{\mathrm{h}}$ is determined according to an analytical model proposed by Uibel and Bla $\beta$ [35] and presented in Eq. (10).

$f_{\mathrm{h}}=0.13 \rho^{1.05} d^{-0.53}$

\subsection{Stiffness properties}

Calculation methods capable of predicting the modulus of subgrade reaction of timber $k_{\mathrm{h}}$ and the withdrawal stiffness of a nail $K_{\mathrm{ax}}$ have not yet been derived. In this study, those stiffness properties are determined based on the test data published in literature.

The modulus of subgrade reaction $k_{\mathrm{h}}$ depends on the diameter of the fastener and the load-to-grain angle $[40,41,46]$. Values of $k_{\mathrm{h}}$ may depend also on the average density of the timber panel; however, very few research projects focused on the modulus of subgrade reaction of timber and limited experimental evidence exists. Consequently, the influence of this last parameter is neglected. In this study, the following values are adopted: if $d$ is greater than or equal to $12 \mathrm{~mm}, k_{\mathrm{h}}=25 \mathrm{MPa} / \mathrm{mm}$ parallel to grain and $k_{\mathrm{h}}=10 \mathrm{MPa} / \mathrm{mm}$ in the orthogonal direction are assumed. If $d$ is smaller than $12 \mathrm{~mm}$ (e.g. a nail), reduced values of $15 \mathrm{MPa} / \mathrm{mm}$ and $10 \mathrm{MPa} / \mathrm{mm}$ can be assumed, respectively.

The withdrawal stiffness of a nail $K_{\mathrm{ax}}$ is determined based on the experimental results published by Izzi et al. [49] and is set to $1250 \mathrm{~N} /$ $\mathrm{mm}$. Such stiffness property depends on both the average density of CLT and the diameter of the fastener. However, due to the lack of analytical expressions capable of predicting it, the experimental value is adopted and its influence on the load-displacement response of the joint is examined.

\section{Numerical analyses}

Simulations focus on the mechanical behaviour of laterally loaded steel-to-timber joints with annular-ringed shank nails in CLT (Fig. 6). Analyses are carried out in two phases: firstly, the behaviour of the model is validated by comparing the numerical predictions of the lateral dowel capacity to the analytical values assessed using the Hilson [36] model. The mechanical behaviour under monotonic and cyclic conditions is analysed afterwards; shear tests are reproduced in parallel and perpendicular to the face lamination of a CLT panel and results are compared to the test data obtained by Izzi et al. [49] on similar joints.

The discussion presented below considers nailed joints with thick plates. According to Eurocode 5 [6], the situation of 'thick plate' is achieved when the thickness of the metal member is greater than the diameter of the nail. However, annular-ringed shank nails have a conical-shaped cap (Fig. 7) that enforces a failure mechanism with two plastic hinges even with thinner plates [49].

\subsection{Mechanical validation of the model}

The mechanical behaviour of the proposed model is validated by comparing the numerical predictions of the lateral dowel capacity to the analytical values determined according to the Hilson [36] model (Eq. (11)). Eq. (11) is based on a plastic limit analysis and is derived assuming that the joint transfer solely shear, neglecting any other parameter as the deformations. Based to this simplification, the withdrawal behaviour of the fastener and the embedding of the timber fibres into the thread are disregarded.
Table 1

Typical input parameters used to model the bending behaviour of the nail (obtained assuming $d=4 \mathrm{~mm}$ ).

\begin{tabular}{ll}
\hline & Input parameter \\
\hline$M_{\mathrm{y}}[\mathrm{Nmm}]$ & 8235 \\
$K_{\Omega}\left[\mathrm{Nmm} /{ }^{\circ}\right]$ & 82,350 \\
Slope \#1, \#10 & $K_{\Omega}$ \\
Slope \#3, \#4 & $K_{\Omega}$ \\
Separation \#3-\#5 & $2 \% \times M_{\mathrm{y}}$ \\
Separation \#5-\#30 & 0
\end{tabular}

$$
F_{\text {lat }}=\min \left\{\begin{array}{cc}
f_{\mathrm{h}} t_{1} d & \text { (a) } \\
f_{\mathrm{h}} t_{1} d\left[\sqrt{2+\frac{4 M_{\mathrm{y}}}{f_{\mathrm{h}} t_{1}^{2}}}-1\right] & \text { (b) } \\
2.0 \sqrt{M_{\mathrm{y}} f_{\mathrm{h}} d} & \text { (c) }
\end{array}\right.
$$

Simulations consider the reference nail introduced in Section 3 and two additional nails with $d=6.0 \mathrm{~mm}$ and $d=8.0 \mathrm{~mm}$, respectively. The pointside penetration depth is kept constant in all the systems analysed $\left(t_{1}=54 \mathrm{~mm}\right)$. The nails are assumed embedded in the first two board layers of a CLT element, as schematized in Fig. 6.

Analyses are performed by varying the discretization of the nail shank and the average density of CLT. Firstly, a coarse discretization is considered, with the nail shank divided into six beams of length $l=9 \mathrm{~mm}$ : three beams are located into the upper board layer and the other three into the lower one. A finer discretization is taken into account afterwards, with the shank divided into nine beams $(l=6 \mathrm{~mm})$ : five beams are located into the upper board layer and four in the lower one, respectively. In both cases, the average density of CLT is varied between $360 \mathrm{~kg} / \mathrm{m}^{3}$ and $500 \mathrm{~kg} / \mathrm{m}^{3}$, with $20 \mathrm{~kg} / \mathrm{m}^{3}$ steps. Tables 1 and 2 list the typical input parameters used in the analyses; values are obtained considering the finer discretization $(l=6 \mathrm{~mm})$, a nail with $d=4.0 \mathrm{~mm}$ and an average density $\rho=480 \mathrm{~kg} / \mathrm{m}^{3}$.

Results of this parametric study are summarized in Fig. 8. Simulations are carried out in displacement control, parallel to the superficial lamination of the panel. The lateral dowel capacity is equal to the load attained by the nailed joint when the plastic deformed configuration is reached. Numerical predictions (red markers) provide a good match with the analytical values determined using Eq. (11) (black lines with markers), especially when the finer discretization is adopted (Fig. 8b). Analyses with $d=4.0 \mathrm{~mm}$ exhibit a failure mechanism where the bending capacity of the nail is attained with two plastic hinges together with embedding of timber. Simulations performed with larger diameters lead to a failure mechanism where only one plastic hinge is attained.

\subsection{Simulations under monotonic loading conditions}

The load-displacement response of the nailed steel-to-timber joint schematized in Fig. 6 is analysed. Monotonic shear tests are reproduced

Table 2

Typical input parameters used to model the withdrawal behaviour of the nail (obtained assuming $d=4 \mathrm{~mm}, \rho=480 \mathrm{~kg} / \mathrm{m}^{3}, l_{\mathrm{thr}}=44 \mathrm{~mm}$, $n=9$, and $l=6 \mathrm{~mm}$ )

\begin{tabular}{ll}
\hline & Input parameter \\
\hline$K_{\text {ax,fastener }}[\mathrm{N} / \mathrm{mm}]$ & 1250 \\
$F_{\mathrm{ax}, \text { fastener }}[\mathrm{N}]$ & 2188 \\
$K_{\mathrm{ax}}[\mathrm{N} / \mathrm{mm}]$ & 139 \\
$F_{\mathrm{ax}}[\mathrm{N}]$ & 243 \\
Slope \#1 & $K_{\mathrm{ax}}$ \\
Slope \#10 & $10 \times K_{\mathrm{ax}}$ \\
Slope \#3, \#4 & $5 \times K_{\mathrm{ax}}$ \\
Separation \#3-\#5 & $2 \% \times F_{\mathrm{ax}}$ \\
Separation \#5-\#10 & 0 \\
\hline
\end{tabular}



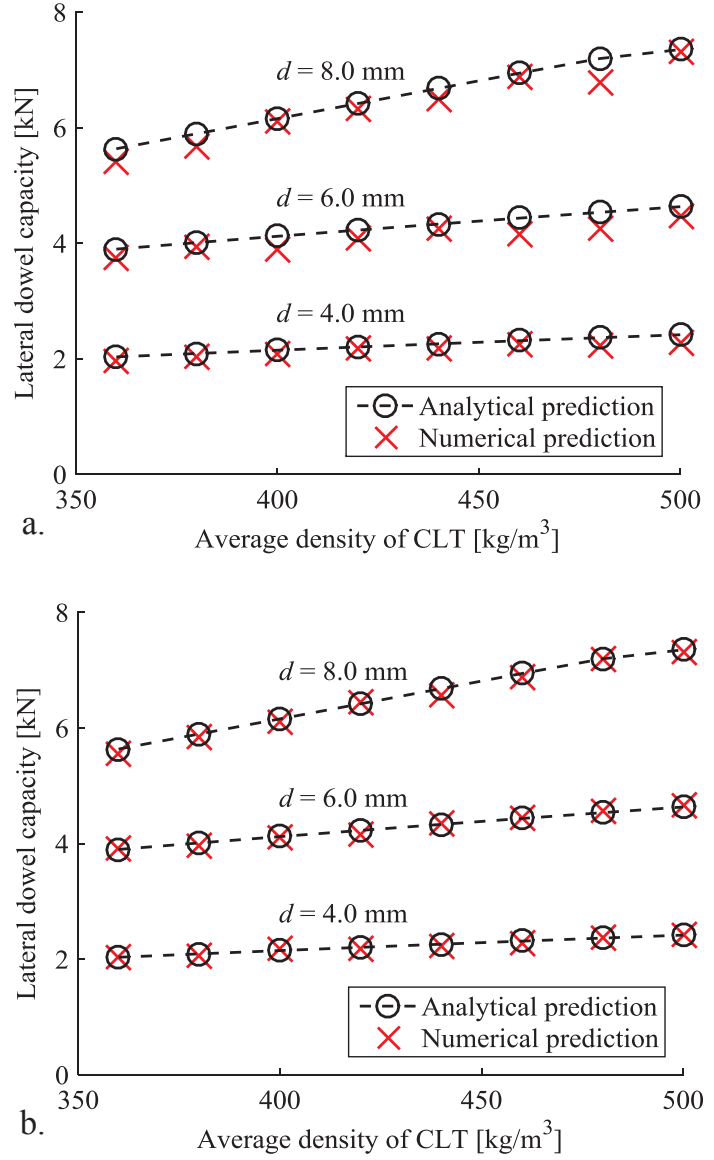

Fig. 8. Lateral dowel capacity of a nailed steel-to-timber joint determined according to the Hilson [36] model (black dashed lines with markers) and using the numerical model (red markers): (a) shank discretized into six beams of length $l=9 \mathrm{~mm}$ and (b) shank discretized into nine beams of length $l=6 \mathrm{~mm}$. (For interpretation of the references to colour in this figure legend, the reader is referred to the web version of this article.)

in parallel and perpendicular to the face lamination of a CLT panel and results are compared to the envelope curves extracted from thirty cyclic shear tests carried out by Izzi et al. [49] (fifteen tests parallel to the face lamination of a CLT panel and fifteen perpendicular, respectively).

Analyses are carried out by assuming that the nail is embedded into the first two board layers of a CLT element with $30 \mathrm{~mm}$ thick boards. The average density of the CLT panel, acquired from the tests, is $\rho=480 \mathrm{~kg} / \mathrm{m}^{3}$.

Based on the model validation (Section 4.1), the nail shank is divided into nine beams of length $l=6 \mathrm{~mm}$ : five beams are located in the upper board layer and four in the lower one. The input parameters used in the analyses are listed in Tables 1, 2 and 3.

Table 3

Typical input parameters used to model the embedment behaviour of timber (obtained assuming $d=4 \mathrm{~mm}, \rho=480 \mathrm{~kg} / \mathrm{m}^{3}$, and $l=6 \mathrm{~mm}$ ).

\begin{tabular}{lll}
\hline & \multicolumn{2}{l}{ Input parameter } \\
\cline { 2 - 3 } & Parallel grain & Perpendicular grain \\
\hline$k_{\mathrm{h}}[\mathrm{MPa} / \mathrm{mm}]$ & 10 & 15 \\
$F_{\mathrm{h}}[\mathrm{MPa}]$ & 41 & 41 \\
$K_{\mathrm{h}}[\mathrm{N} / \mathrm{mm}]$ & 240 & 360 \\
$F_{\mathrm{h}}[\mathrm{N}]$ & 984 & 984 \\
Slope \#1, \#10 & $K_{\mathrm{h}}$ & $K_{\mathrm{h}}$ \\
Slope \#3 & $5 \times K_{\mathrm{h}}$ & $5 \times K_{\mathrm{h}}$ \\
Slope \#4 & $2.5 \times K_{\mathrm{h}}$ & $2.5 \times K_{\mathrm{h}}$ \\
Separation \#3-\#5 & $2 \% \times F_{\mathrm{h}}$ & $2 \% \times F_{\mathrm{h}}$ \\
Separation \#50-\#4 & $2 \% \times F_{\mathrm{h}}$ & $2 \% \times F_{\mathrm{h}}$ \\
\hline
\end{tabular}
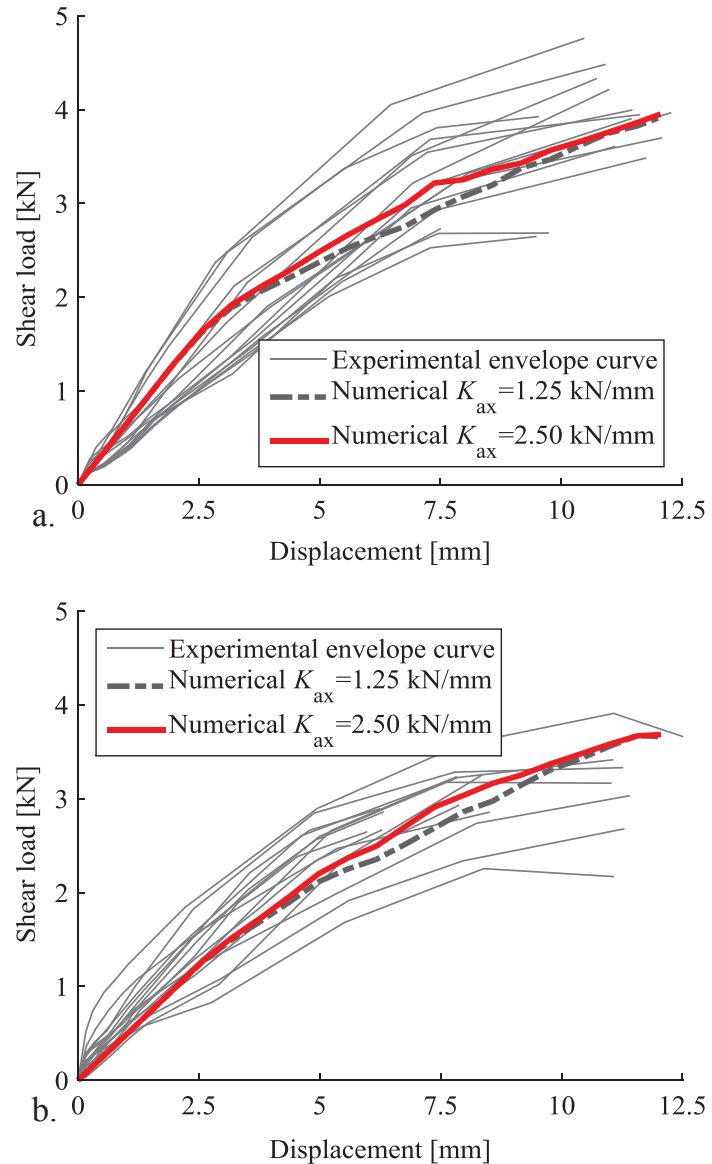

Fig. 9. Comparison between experimental shear tests (grey solid lines) and numerical results, obtained by considering $K_{\mathrm{ax}}=1.25 \mathrm{kN} / \mathrm{mm}$ (actual withdrawal stiffness of the nail, dark grey dashed line) and $K_{\mathrm{ax}}=2.50 \mathrm{kN} / \mathrm{mm}$ (red line): (a) parallel and (b) perpendicular to the face lamination of a CLT panel. (For interpretation of the references to colour in this figure legend, the reader is referred to the web version of this article.)

The first set of simulations neglects the embedding of the timber fibres into the thread of the nail. Analyses are subsequently repeated and this effect is introduced by increasing the withdrawal stiffness of the nail. Consequently, according to Domínguez et al. [47] and based on the profile of the shank, the withdrawal stiffness is doubled up.

The outcome of this study is shown in Fig. 9, where experimental results (grey solid lines) and numerical predictions determined considering the original withdrawal stiffness (dark grey dashed line) and the doubled one (red line) are compared. Simulations show that the lateral dowel capacity of the joint controls the bearing mechanism up to $3 \mathrm{~mm}$ of displacement, while the withdrawal behaviour of the nail is activated afterwards. Results obtained with the doubled withdrawal stiffness lead to slightly better performances after the attainment of the lateral dowel capacity, even though the maximum load-carrying capacity is similar to the original simulations. Based on this behaviour, the embedding of the timber fibres into the thread of the shank is neglected in the cyclic simulations.

\subsection{Simulations under cyclic loading conditions}

The hysteretic behaviour and the energy dissipation of the joint schematized in Fig. 6 are analysed. Cyclic shear tests are reproduced in parallel and perpendicular to the face lamination of a CLT panel and numerical results are compared to two tests carried out by Izzi et al. [49]. Analyses considered the same input parameters adopted in Section 4.2 and the displacement histories of the tests.

The outcome of this study is shown in Fig. 10, where experimental 

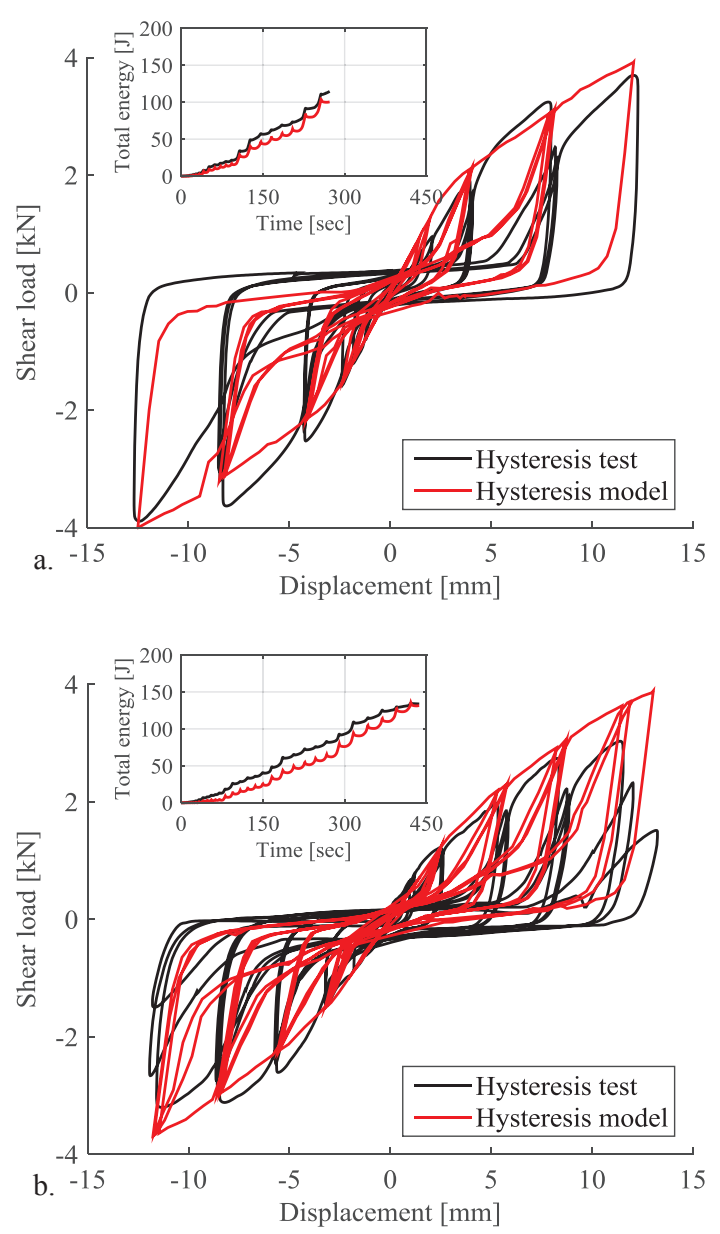

Fig. 10. Comparison between experimental hysteresis tests (black line) and numerical results (red line): (a) parallel to and (b) perpendicular to the face lamination of a CLT panel (with close-up on the history of total energy). (For interpretation of the references to colour in this figure legend, the reader is referred to the web version of this article.)

(black solid line) and numerical results (red solid line) are compared. Analyses provide a good match with the reference test results in terms of both global shape of the hysteresis cycles and total energy dissipation, leading to a final difference lower than $5 \%$. However, there are small discrepancies that might be minimized by further refining the mechanical calibration of the embedment behaviour of timber. This applies to both the reloading stiffness (the slope of branch \#4 in Fig. 4c) and the lack of impairment of mechanical properties due to cyclic loading. The reloading stiffness is determined based on the results of Chui and Ni [46] (Section 2.3), which were obtained employing nails with diameter smaller than the those considered in this numerical study. The strength and stiffness degradations, although implemented into the subroutine used in the simulations, have not been considered since require the assessment of additional input parameters for which no experimental evidence exists. Therefore, to improve the reliability of the results, the future developments of this study should overcome this lack of knowledge and perform cyclic embedment tests using nails with diameter greater than or equal to $4 \mathrm{~mm}$.

\section{Conclusions}

This paper proposes a numerical model capable of predicting the hysteretic behaviour and failure mechanisms of timber joints with dowel-type fasteners. A key feature of the model is the use of simplified mechanical relationships and basic input parameters that can be assessed using either experimental approaches or the information available in literature. This allows performing parametric numerical studies where the type and diameter of the fastener, the density of timber, and the load-to-grain angle are varied.

The model schematizes a timber joint as an elasto-plastic beam in a non-linear medium with a compression-only behaviour. The behaviour of the steel and timber components of the joint is modelled using nonlinear springs, characterized by elasto-plastic constitutive laws. Moreover, the model is capable of simulating the effect of the cavity formed around the fastener by timber crushing, and predicts both the hysteretic behaviour and the energy dissipation under cyclic loading conditions.

Shear tests are reproduced on steel-to-timber joints with annularringed shank nails in CLT and results are compared to the experimental test data obtained on similar elements. Analyses conducted under monotonic conditions highlighted that the bearing mechanism is governed by the lateral dowel capacity of the joint up to $3 \mathrm{~mm}$ of displacement, while the withdrawal behaviour of the nail is activated afterwards. Simulations carried out under cyclic conditions lead to sufficiently accurate predictions of the mechanical behaviour (in terms of elastic stiffness, maximum load-carrying capacity, and energy dissipation), even though the reloading branches of the hysteresis cycles exhibited small discrepancies with the reference tests. Differences can be attributed to the simplifications introduced in the analyses to model the embedment behaviour of timber under cyclic loads.

The numerical model proposed in this paper represents a sound basis to investigate the hysteretic behaviour of joints with dowel-type fasteners. Nevertheless, future research is required to further verify and improve this predictive model. Extending the knowledge on the embedment behaviour of timber under cyclic loads represents a matter of primary importance to improve the reliability of the numerical results. Most of the results currently available in literature are obtained under monotonic conditions and considering fasteners with diameter greater than $10 \mathrm{~mm}$. To this aim, it would be useful to design and perform test programmes considering fastener with smaller diameters (with $d$ smaller than $8 \mathrm{~mm}$ ). A second recommendation for future developments deals with the extension of the current numerical study to other configurations of technical interests. The study presented in this paper focused on the mechanical behaviour of nailed joints for CLT structures; however, the method proposed in Section 2 and 3 might be used to develop numerical models that reproduce sheathing-to-framing joints for light timber-frame structures, as well as timber-to-timber and slotted-in steel plate joints in solid wood.

\section{Acknowledgments}

The experimental results taken as a reference in this study were obtained in the framework of two Short Term Scientific Missions at the Institute of Timber Engineering and Wood Technology, Graz University of Technology (Graz, Austria). The STSMs were supported by the COST Actions FP1004 and FP1402, which are gratefully acknowledged. Professor Gerhard Schickhofer and Dr. Georg Flatscher from Graz University of Technology are acknowledged for their help in the planning, preparation and execution of the tests.

\section{References}

[1] Filiatrault A, Christovasilis IP, Wanitkorkul A, Van de Lindt JW. Experimental seismic response of a full-scale light-frame wood building. J Struct Eng 2010;136(3):246-54. http://dx. doi.org/10.1061/(ASCE)ST.1943-541X.0000112.

[2] Van de Lindt JW, Pei S, Pryor SE, Shimizu H, Isoda H. Experimental seismic response of a full-scale six-story light-frame wood building. J Struct Eng 2010;136(10):1262-72. http://dx.doi.org/10.1061/(ASCE)ST.1943-541X. 0000222.

[3] Ceccotti A, Sandhaas C, Okabe M, Yasumura M, Minowa C, Kawai N. SOFIE project 3D shaking table test on a seven-storey full-scale cross-laminated building. Earthquake Eng Struct Dyn 2013;42(13):2003-21. http://dx.doi.org/10.1002/eqe. 2309.

[4] Flatscher G, Schickhofer G. Shaking-table test of a cross-laminated timber structure. Proc ICE - Struct Build 2015;168(11):878-88. http://dx.doi.org/10.1680/stbu.13. 00086. 
[5] Tomasi R, Casagrande D, Grossi P, Sartori T. Shaking table tests on a three-storey timber building. Proc ICE - Struct Build 2015;168(11):853-67. http://dx.doi.org/ $10.1680 /$ jstbu.14.00026.

[6] EN 1995-1-1:2004/A2. Eurocode 5: design of timber structures. Part 1-1: General. Common rules and rules for buildings. CEN, Brussels, Belgium; 2014.

[7] Johansen KW. Theory of timber connections. Int Assoc Bridge Struct Eng 1949;9:249-62. http://dx.doi.org/10.5169/seals-9703.

[8] Ivanov IM. The new measure of the strength of wood and methods of its determi nation. Bull Forest Inst Acad Sci USSR 1949;4:34-45.

[9] Mack JJ. The strength and stiffness of nailed joints under short-duration loading. Division of forest products, technological paper n. 40, Commonwealth Scientific and Industrial Research Organization, Melbourne Australia; 1966

[10] Kuenzi EW. Theoretical design of nailed or bolted joint under lateral load. United States Department of Agriculture, Forest Service, Forest Laboratory Products, Report No: D1951 (revised March 1955), Madison, Wisconsin, USA; 1955.

[11] Chui YH, Ni C, Jiang L. Finite-element model for nailed wood joints under reversed cyclic load. J Struct Eng 1998;124(1):96-103. http://dx.doi.org/10.1061/(ASCE) 0733-9445(1998) 124:1(96).

[12] Foschi RO. Modeling the hysteretic response of mechanical connections for wood structures. In: World Conference on Timber Engineering (WCTE), Whistler, British Columbia, Canada; 2000.

[13] Li M, Foschi RO, Lam F. Modeling hysteretic behavior of wood shear walls with a protocol-independent nail connection algorithm. J Struct Eng 2012;138(1):99-108. http://dx.doi.org/10.1061/(ASCE)ST.1943-541X.0000438.

[14] Plesnik T, Erochko J, Doudak G. Nailed connection behavior in light-frame wood shear walls with an intermediate layer of insulation. J Struct Eng 2016;142(7). 04016045(1-9), doi: 10.1061/(ASCE)ST.1943-541X.0001506.

[15] Foliente GC. Hysteresis modeling of wood joints and structural systems. J Struct Eng 1995;121(6):1013-22. http://dx.doi.org/10.1061/(ASCE)0733-9445(1995) 121:6(1013).

[16] Folz B, Filiatrault A. Cyclic analysis of wood shear walls. J Struct Eng 2001;127(4):433-41. http://dx.doi.org/10.1061/(ASCE)0733-9445(2001) 127:4(433).

[17] He M, Lam F, Foschi RO. Modeling three-dimensional timber light-frame buildings. J Struct Eng 2001;127(8):901-13. http://dx.doi.org/10.1061/(ASCE)07339445(2001) 127:8(901).

[18] Judd JP, Fonseca FS. Analytical model for sheathing-to-framing connections in wood shear walls and diaphragms. J Struct Eng 2005;131(2):345-52. http://dx.doi. org/10.1061/(ASCE)0733-9445(2005) 131:2(345).

[19] Xu J, Dolan JD. Development of nailed wood joint element in ABAQUS. J Struct Eng 2009;135(8):968-76. http://dx doi org/10.1061/ASCE ST.1943-541X.0000030.

[20] Rinaldin G, Amadio C, Fragiacomo M. A component approach for the hysteretic behaviour of connections in cross-laminated wooden structures. Earthquake Eng Struct Dyn 2013;42(13):2023-42. http://dx.doi.org/10.1002/eqe.2310.

[21] Pozza L, Saetta A, Savoia M, Talledo D. Coupled axial-shear numerical model for CLT connections. Constr Build Mater 2017;150:568-82. http://dx.doi.org/10. 1016/j.conbuildmat.2017.05.141.

[22] Santos CL, De Jesus AMP, Morais JJL, Lousada JLPC. Quasi-static mechanical be haviour of a double-shear single dowel wood connection. Constr Build Mater 2008;23:171-82. http://dx.doi.org/10.1016/j.conbuildmat.2008.01.005.

[23] González Fueyro JL, Domínguez M, Cabezas JA, Cabezas MP. Design of connections with metal dowel-type fasteners in double shear. Mater Struct 2009;42(3):385-97. http://dx.doi.org/10.1617/s11527-008-9389-3.

[24] Hong J-P, Barrett D. Three-dimensional finite-element modeling of nailed connections in wood. J Struct Eng 2010;136(6):715-22. http://dx.doi.org/10.1061/ASCF ST.1943-541X.0000160.

[25] Meghlat EM, Oudjene M, Ait-Aider H, Batoz JL. A new approach to model nailed and screwed timber joints using the finite element method. Constr Build Mater 2013:41:263-9. http://dx.doi.org/10.1016/j.conbuildmat.2012.11.068.

[26] Oudjene M, Meghlat EM, Ait-Aider H, Batoz JL. Non-linear finite element modelling of the structural behaviour of screwed timber-to-concrete composite connections. Compos Struct 2013;102:20-8. http://dx.doi.org/10.1016/j.compstruct.2013.02. 007.

[27] Sandhaas C. Material model for wood. Heron 2013; 53(2-3): 179-200 [Stevin Lab. and Inst. TNO for Bldg Materials and Structures, Delft, The Netherlands].

[28] Fahrni R. Finite-element modeling of timber connections MS Thesis Zürich, Schweiz: ETH Zurich; 2016.

[29] Hassanieh A, Valipour HR, Bradford MA, Sandhaas C. Modelling of steel-timber composite connections: validation of finite element model and parametric study. Eng Struct 2017;138:35-49. http://dx.doi.org/10.1016/j.engstruct.2017.02.016.

[30] EN 409. Timber structures. Test methods. Determination of the yield moment of dowel type fasteners. CEN, Brussels, Belgium; 2009.

[31] EN 383. Timber structures. Test methods. Determination of embedment strength and foundation values for dowel type fasteners. CEN, Brussels, Belgium; 2007.

[32] EN 1382. Timber structures. Test methods. Withdrawal capacity of timber fasteners. CEN, Brussels, Belgium; 2016.

[33] Sandhaas C, Mergny E. Yield moment of nails. INTER 2016 Meeting, Graz, Austria, Paper Note $1 ; 2016$

[34] Blaß HJ, Uibel T. Tragfähigkeit von stiftförmigen Verbindungsmitteln in Brettsperrholz. 8, Karlsruher Berichte zum Ingenieurholzbau, Karlsruhe, Germany; 2007. doi: http://dx.doi.org/10.5445/KSP/1000006318.

[35] Uibel T, Blaß HJ. Load carrying capacity of joints with dowel type fasteners in solid wood panels. In: 39th CIB-W18 Meeting, Florence, Italy; 2006. Paper 39-7-5.

[36] Hilson BO. Joints with dowel-type fasteners - theory. Timber engineering STEP 1: basis of design, material properties, structural components and joints. Centrum Hout, Almere, The Netherlands; 1995. p. C3/1-11.

[37] Simulia DS. ABAQUS 6.12 documentation. Rhode Island, USA: Providence; 2012.

[38] Rinaldin G, Amadio C, Macorini L. A macro-model with nonlinear springs for seismic analysis of URM buildings. Earthquake Eng Struct Dyn 2016;45(14):2261-81. http://dx.doi.org/10.1002/eqe.2759.

[39] Rinaldin G. Modellazione e analisi non lineare di strutture in muratura e legno PhD Thesis Trieste, Italy: University of Trieste; 2012

[40] Karagiannis V, Málaga-Chuquitaype C, Elghazouli AY. Modified foundation modelling of dowel embedment in glulam connections. Constr Build Mater 2016;102:1168-79. http://dx.doi.org/10.1016/j.conbuildmat.2015.09.021.

[41] Pedersen MU. Dowel type timber connections - strength modelling PhD Thesis Copenhagen, Denmark: Technical University of Denmark; 2002

[42] Sewata K, Yasumura M. Determination of embedding strength of wood for doweltype fasteners. J Wood Sci 2002;48(2):138-46. http://dx.doi.org/10.1007/ BF00767291.

[43] Blaß HJ, Schädle P. Ductility aspects of reinforced and non-reinforced timber joints. Eng Struct 2011;33(11):3018-26. http://dx.doi.org/10.1016/j.engstruct.2011.02. 001.

[44] Glišović I, Boško S, Tatjana K-M. Embedment test of wood for dowel-type fasteners Wood Res 2012;57(4):639-50.

[45] Zhou T, Guan Z. Review of existing and newly developed approaches to obtain timber embedding strength. Prog Struct Mater Eng 2006;8(2):49-67. http://dx.doi. org/10.1002/pse.213.

[46] Chui YH, Ni C. Load-embedment response of timber to reversed cyclic load. Wood Fiber Sci 1997;29(2):148-60.

[47] Domínguez M, González Fueyro JL, Cabezas JA. Accounting of the thread embedment in timber structures dowel-type joints. Load-slip relationship. Proc Inst Mech Eng, Part C: J Mech Eng Sci 2017;231(1):150-60. http://dx.doi.org/10.1177/ 0954406216663578.

[48] ETA-04/0013. European Technical Assessment. Nails and screws for use in nailing plates in timber structures. ETA-Denmark, Nordhavn, Denmark; 2015.

[49] Izzi M, Flatscher G, Fragiacomo M, Schickhofer G. Experimental investigations and design provisions of steel-to-timber joints with annular-ringed shank nails for CrossLaminated Timber structures. Constr Build Mater 2016;122:446-57. http://dx.doi. org/10.1016/j.conbuildmat.2016.06.072. 